\title{
Nuclear data research supported by EURATOM: CHANDA, ERINDA and EUFRAT
}

\author{
Enrique Miguel Gonzalez ${ }^{1, *}$, Arnd Rudolf Junghans ${ }^{2}$, Arjan Plompen ${ }^{3}$, and Peter Schillebeeckx ${ }^{3}$ \\ ${ }^{1}$ Centro de Investigaciones Energéticas, Medioambientales y Tecnológicas (CIEMAT), Avda. Complutense, 40,28040 Madrid, \\ Spain \\ ${ }^{2}$ Helmholtz-Zentrum Dresden - Rossendorf (HZDR), Bautzner Landstr. 400, 01328 Dresden, Germany \\ 3 Joint Research Centre (JRC), Retieseweg 111, 2440 Geel, Belgium
}

Received: 12 March 2019 / Accepted: 4 June 2019

\begin{abstract}
Nuclear data and associated tools are critical elements of the nuclear energy industry and research, playing an essential role in the simulation of nuclear systems, safety and performance calculations and interpretation of the reactor instrumentation. Nuclear data improvement requires a combination of much different know-hows that are distributed over many small- and medium-sized institutions along Europe. The Euratom programs have facilitated the setup of pan European collaborations getting together the required experience inside the projects CHANDA, ERINDA and the JRC action EUFRAT. The paper describes the holistic and inclusive approach of these projects that have also worked together to coordinate the European nuclear data research capabilities to improve the facilities, detectors, models and evaluation, validation and simulation tools. It also shows examples of success histories and summary of results of these projects and of their impact on the EU nuclear safety and industry, together with an outlook to the future.
\end{abstract}

\section{Introduction}

Nuclear data and associated tools are a critical element of the nuclear energy industry and research. They play an essential role in the simulation of nuclear systems or devices for nuclear energy and non-energy applications, for the calculation of safety and performance parameters of existing and future reactors and other nuclear facilities, for the innovation of the design of those nuclear facilities and the innovation on radioactive devices and use of radioactive materials in non-energy applications, and for the interpretation of measurements in these facilities and systems.

Nuclear data, ND, is often not visible for applications that rely on the huge data sets of nuclear cross sections, emission probabilities, branching ratios, atomic masses, life times, energy levels, fission yields and many other nuclear data. However, with the present computing power and the development of the simulation codes, in many cases the limiting factor for the accuracy and prediction capabilities of these simulation codes comes from the accuracy of the relevant nuclear data and their uncertainties. Indeed, no

\footnotetext{
* e-mail: enrique.gonzalez@ciemat.es
}

matter how sophisticated the tool is, no simulation, calculation or interpretation of measurements can be better than the limit imposed by the nuclear data they use.

For these reasons, there are continuous request of new or better nuclear data, coming from new levels of safety, new safety criteria and scenarios, new reactor designs or new applications or new modes of operations of present reactors, innovative solutions for waste management and from pending requests, not feasible in the past, that can be addressed with the present R\&D on nuclear data and tools. These requests are regularly evaluated and maintained in high-priority request lists, in the framework of international initiatives and international organisation like IAEA and NEA/OECD.

In order to have nuclear data available to applications, several steps are needed in what is known as the nuclear data cycle. Nuclear data are typically deduced from differential (microscopic) measurements (a more or less direct measurement of the reaction of interest separated from other effects). This requires preparation of a high purity sample of the nuclide to measure, often radioactive and scarce, as well as the availability of sophisticated detection systems and controllable sources of neutrons and other radiations (often based on particle accelerators). Then the data are analyzed and the results are provided to international databases. Putting together results of several measurements and using nuclear theories, the data are 
further analyzed, and finally assembled into what is known as "evaluated nuclear data libraries". These evaluated data are then validated by comparing their predictions to integral experiments (complex systems, typically experimental reactors). From the differences between predictions and integral experiments, we can deduce corrections to the basic nuclear data and develop better evaluated libraries. This validation process can also reveal a possible need for additional differential measurements or evaluations, repeating the process until the required accuracy is achieved.

As a consequence, producing high quality data requires a combination of much different know-hows (target production, detectors, neutron sources, analysis, evaluation, nuclear theory, nuclear reactors, simulation codes, and others). In addition, it is important to realize that the necessary expert know-how is widely distributed within many research teams, particularly in Europe, and that most of these teams specialize only on one or few components of the nuclear data cycle. Therefore, in order to provide the nuclear data needed, it is important to prepare a very well structured wide and well synchronized collaboration between the key EU expert institutions.

The EURATOM framework program has been instrumental during the FP7 and before, to nucleate panEuropean collaborations of laboratories that on one side have developed competitive projects to develop the tools and perform measurements, evaluation and validation of new or improved nuclear data like CHANDA. It has also facilitated the setup of frameworks for easy and efficient transnational access to experimental facilities needed for those activities, like the competitive proposal ERINDA and the direct JRC action EUFRAT.

\subsection{ERINDA}

The ERINDA project [1] (European Research Infrastructures for Nuclear Data Applications) has coordinated the European efforts to exploit up-to-date neutron beam technology for novel research on advanced concepts for nuclear fission reactors and the transmutation of radioactive waste. For the development of these transmutation systems and for improved nuclear safety, accurate nuclear data haven been obtained in the ERINDA project. The strategic objectives of ERINDA were:

- to provide transnational access for nuclear data measurements at the consortium's facilities;

- experiments should account for nuclear data requests of highest priority and scientific value;

- improve simulation methods to predict the running conditions of innovative reactor systems and the transmutation of nuclear waste;

- generation of complete, accurate and consistent nuclear data libraries and measured nuclear reaction crosssections.

ERINDA offered the nuclear data research infrastructures of 13 partners (HZDR, JRC-GEEL, CERN, CENBG, IPNO, UU-TSL, PTB, NPI, IKI, IFIN-HH, NPL, FRANZ and CEA) from all over Europe to experimental teams making new nuclear data measurements. The ERINDA facilities included different neutron sources and methods for nuclear data measurement, in particular: - time of flight facilities for fast neutrons:

- nELBE (HZDR, Dresden); n_TOF (CERN, Geneva); GELINA (JRC, Geel);

- charged-particle accelerators:

- production of quasi-monoenergetic neutrons electrostatic accelerators in Bordeaux, Orsay, Bucharest and Dresden,

- neutron reference fields at PTB Braunschweig and NPL Teddington,

- cyclotrons in Řě̌, Jyväskylä, Oslo and Uppsala with neutron energy range up to $180 \mathrm{MeV}$,

- pulsed proton linear accelerator in Frankfurt;

- research reactors:

- Budapest and Řě cold neutron beam, Prompt Gamma Activation Analysis.

Within the project 3015 additional hours of beam time at the consortium facilities have been provided in 26 experiments as transnational-access including technical and travel support for the user groups. In addition, 16 short term visits (with a total duration of 106 weeks) of scientists to the consortium institutes were supported. In this way theoretical data analysis and computer simulations relevant to the experiments were performed. All ERINDA facilities were grouped in a pool. To optimize the scientific output of the experiments, a Project Advisory Committee (PAC) consisting of five external experts selected from the submitted experiment proposals was made and decided about the best suited facility for a certain type of measurement. The transnational access budget was distributed according to the PAC decisions. The participation of post-doctoral fellows and $\mathrm{PhD}$ students in all ERINDA activities was especially encouraged.

Four European scientific meetings in Dresden, Prague, Jyväskylä and Geneva were organized to communicate the progress and disseminate the results of the ERINDA project.

\subsection{EUFRAT}

Since 2005, JRC-Geel has a programme offering access to its nuclear research infrastructure for external users. In the period 2005-2012 the programme was running with support from DG-RTD (indirect actions NUDAME and EUFRAT). To transform it into a sustainable programme, the open access runs since the beginning of 2014 as an institutional project entitled "European Facilities for Nuclear Reaction and Decay Data Measurements (EUFRAT)". In 2017, EUFRAT [2] was selected as a pilot project to start a JRC-wide open access scheme that includes nuclear and non-nuclear research infrastructures. The JRC-Geel approach for open access to its nuclear facilities has been copied for three other transnational access projects in the nuclear data field that were supported by DG-RTD, i.e. EFNUDAT, ERINDA and CHANDA.

The nuclear research facilities at JRC-Geel are designed for the measurements of highly accurate neutron cross section and nuclear decay data in support to nuclear energy applications: safe operation of nuclear reactors, nuclear safeguards, safe handling of nuclear waste and safe, 
ecological and economical disposal of spent nuclear fuel. They also serve the needs for non-energy applications: production of medical radionuclides, the safety of citizen and environment, environmental tracer studies to understand climate change, new detector developments, nuclear astrophysics, cultural heritage and materials research. The nuclear infrastructure at JRC-Geel includes:

- the GELINA research infrastructure, which combines a white neutron source produced by a $150 \mathrm{MeV}$ linear electron accelerator with a high-resolution neutron timeof-flight facility;

- the MONNET research infrastructure for the production of continuous and pulsed proton-, deuteron- and helium ion beams is based on a 3.5 MV Tandem accelerator and serves for the production of well-characterised quasi mono-energetic neutrons. The tandem replaces the $7 \mathrm{MV}$ Van de Graaff (VdG) accelerator that was operated until August 2015;

- the RADMET radionuclide metrology laboratories, which are used for radioactivity measurements;

- an ultra low-level radioactivity laboratory, which is hosted in the deep-underground facility HADES of the $\mathrm{SCK} \bullet \mathrm{CEN}$; and

- a laboratory for the preparation and characterisation of samples and targets needed for nuclear data measurements.

\subsection{CHANDA}

The CHANDA project [3] brought together the majority of the European nuclear data community, infrastructures and resources to prepare the methodologies, detectors, facilities, interpretation models and tools to produce and use nuclear data with the quality required to comply with the needs for the safety standards that are mandatory for present and future European nuclear reactors and other installations using radioactive materials. Significant technical, methodological and organizational challenges have previously prevented the achievement of this goal for a number of relevant isotopes and nuclear reactions and CHANDA has focused its effort on overcoming those challenges.

CHANDA included 36 partners (CIEMAT, ANSALDO, CCFE, CEA, CERN, CNRS, CSIC, ENEA, GANIL, HZDR, IFIN-HH, INFN, IST-ID, JRC, JSI, JYU, KFKI, NNL, NPI, NPL, NRG, NTUA, PSI, PTB, SCK, TUW, UB, UFrank, UMainz, UMan, UPC, UPM, USC, UU, UOslo, US) from 16 countries from EU plus Switzerland and Norway and 18 of the most relevant facilities equipped to measure nuclear data. The project partners have been strongly involved in previous EURATOM projects producing or using nuclear data and in international organizations dedicated to the compilation, validation and distribution of nuclear data (such as the OECD's Nuclear Energy Agency (NEA/OECD) and the International Atomic Energy Agency (IAEA)) and include most of the participants in FP7 nuclear data projects: ANDES, EUFRAT and ERINDA.

CHANDA was structured in 13 work-packages (WP) organized in four domains of activity. The relations of the different WPs and with external organizations, other projects and the facilities are described in Figure 1. Domain C (DMC) has contributed to upgrade the capacities of the EU nuclear data facilities by development and validation of methodologies of experimental techniques, detection systems, integral measurements, evaluation methods and uncertainty estimation. This domain also produced most of the scientific and technical results like new measurements, new evaluated files and new uncertainty libraries. Domain B has contributed to setup and commission important new experimental facilities and to organize and facilitate transnational access to relevant ND facilities combining support to the facility and to the visiting teams. Domain A (DMA) included the coordination activities, enabling the development of a common vision, of a research roadmap for several years, and of the management structure to make this happen. DMA also included the target fabrication and characterization activities and their organization in the form of a dedicated network. Finally, Domain Management included the project management, but also the coordination of the education and training activities like the preparation of specific courses.

\section{Technical achievements}

The two main characteristics of the ND projects of FP7 were their holistic and inclusive approach. To produce new ND for the final users involves many different steps requiring different facilities and tools. CHANDA has covered all these steps improving the tools and status of each of them but making sure that the improvement is focused on a more efficient preparation of the high priority nuclear data needs. Also, ERINDA and EUFRAT have covered the different types of facilities for the different steps of the ND preparation cycle and have articulated the support both to the facility and the visiting teams to make sure the experiments are successful.

Altogether CHANDA, ERINDA and EUFRAT have contributed to the following elements of the nuclear data preparation.

Improving the facilities: with the help of these EURATOM projects several facilities have improved their experimental conditions for ND experiments, like for example nELBE (HZDR) where the first photoneutron source at a superconducting electron accelerator went into operation, IGISOL (JYU) that was optimized to guide fission fragments into ion-traps, JRC-Geel (JRC), and others. The most significant effort within CHANDA has been on the new experimental area, n_TOF EAR2, for high flux experiments, which allows increasing a factor 30 40 the neutron flux at $\mathrm{n}_{-} \mathrm{TOF}$, and allowing as demonstration the measurement of the ${ }^{7} \operatorname{Be}(n, \alpha)$ reaction cross section using a sample of just $1 \mu \mathrm{g}$ of ${ }^{7} \mathrm{Be}$ [4]. The LICORNE facility at IPN Orsay provides quasi-monoenergetic neutron beam with low background using inverse kinematics with a ${ }^{7} \mathrm{Li}$ beam on a hydrogenous target. The PTB PIAF facility and the JRC-GEEL MONET facilities received new Van de Graaff accelerators for the neutron beam production. 


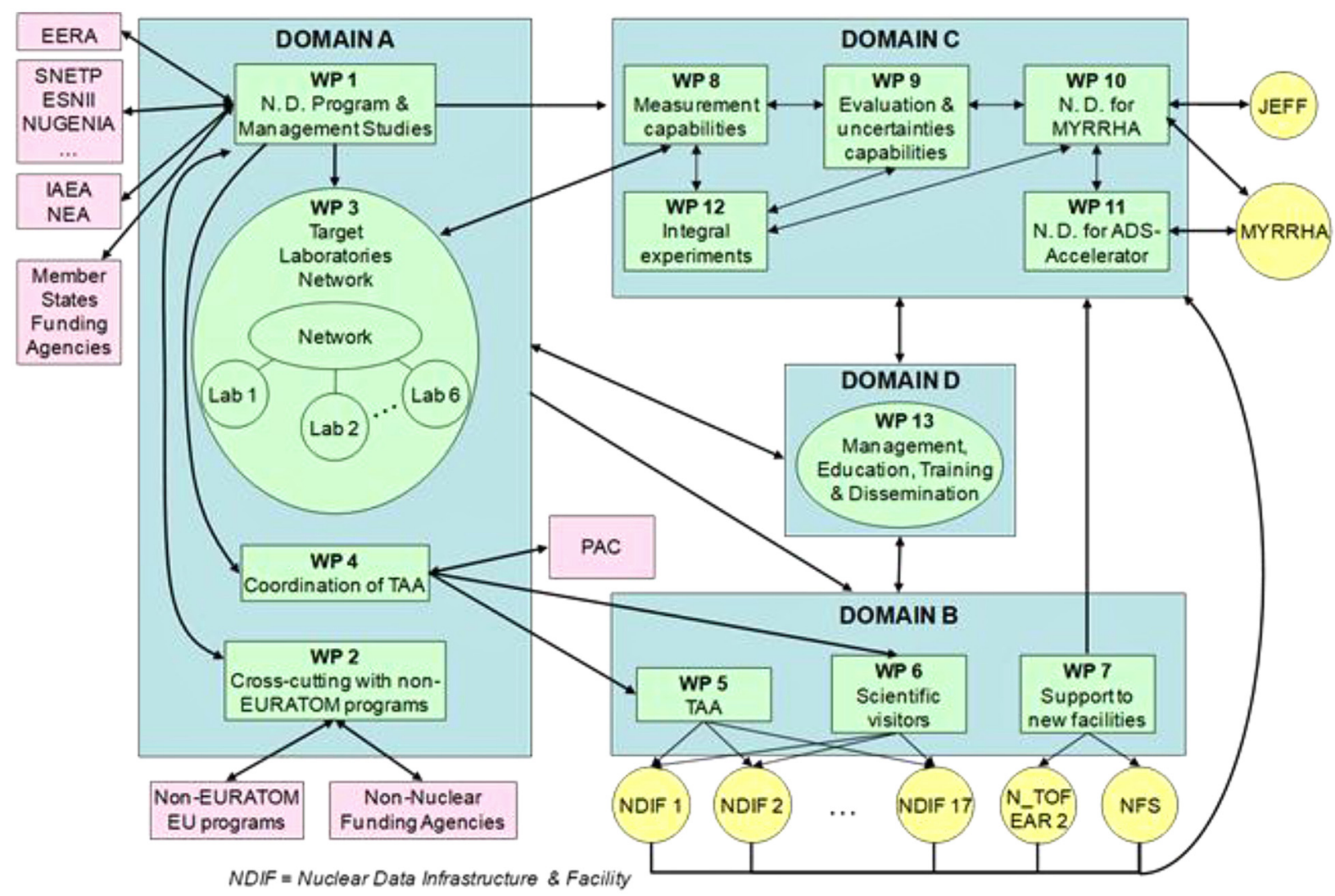

Fig. 1. CHANDA structure of activities and external connections.

Integrating and developing target fabrication capabilities: with improved capabilities on PSI, U Mainz and JRCGeel laboratories. This action helped to better identify and describe the target needed and to actually fabricate 45 very specialized target for ND measurements, most of them highly radioactive and including samples of 10 different actinides.

New methods for cross section measurements: with developments of new detectors (micromegas, DELCO, SCONE, DTAS, BELEN, BRIKEN, FALSTAFF, STEFF), modification of facilities ( $n$ TOF EAR2, AFIRA, GAINS and GRAPhEME at JR $\bar{C}$ ), new combinations of detectors ( $\mathrm{n}$ TOF Total Absorption Calorimeter and a stack of 10 micromegas for capture in fissile actinides), etc.

Comprehensive developments for concurring reactions: making sure that the detector developments, new targets, neutron sources and facilities allow to properly cover the most relevant reactions including capture, fission, inelastic, $(\mathrm{n}, \mathrm{xn}),(\mathrm{n}, \mathrm{chp}), \ldots$

New and improved evaluation models and tools: including the development of TALYS-1.9 that has become the reference European code in evaluation, the improvements of the databases EXFOR and Nuclear Data for Fission Fragments, and the extension of CONRAD.

Systematic and comprehensive uncertainties and correlation libraries in the evaluation: including a complete Bayesian evaluation technique which accounts for model deficiencies in update process and demonstrated with ${ }^{181}$ Ta.
Validation and improvement of data using integral experiments: including the comparison of different uncertainty propagation methods, testing various integral data assimilation methodologies between the "all deterministic" and the "Full Monte-Carlo" methods, and the exchange of samples (Am) between differential (JRC) and integral experiments (MINERVE).

Fast and comprehensive dissemination of results: by close cooperating with responsible agencies, including strong collaboration with IAEA to make sure all relevant experimental results from CHANDA are readily available for evaluators and other users from the EXFOR database for experimental data. Also strong collaboration with $\mathrm{JEFF} / \mathrm{NEA}$ for the incorporation of new data and evaluation tools in the JEFF activities and data libraries, and with large contribution to the CIELO exercise for the update of the most important cross sections and ND for reactor operation. Finally, there has been continuous communication with the NEA High priority request list (HPRL) for nuclear data for progress made and to get updated on the highest priority requests.

Comprehensive tools for transport problems including high energy particles: improvements of existing tools used to simulate experiments or facilities involving high energy particles (above $20 \mathrm{MeV}$ ) to be able to test uncertainty propagation on critical parameters for the safety of MYRRHA like power or spallation yields, improving the reliability of the high-energy nuclear models by comparing them with relevant experimental data (PSI at $590 \mathrm{MeV}$ ) and allowing to explain the deviations on the ${ }^{210} \mathrm{Po}$ 
Table 1. Differential nuclear data measurements carried out within CHANDA.

\begin{tabular}{|c|c|}
\hline$(n, f)$ Cross sections & $(\mathbf{n}, \mathbf{n}),(\mathbf{n}, \mathbf{x n})$ and $\left(\mathbf{n}, \mathbf{n}^{\prime} \gamma\right)$ Cross sections \\
\hline $240,242 \mathrm{Pu}(\mathrm{n}, \mathrm{f})$ & ${ }^{\text {nat }} \mathrm{Fe}(\mathrm{n}, \mathrm{n})$ \\
\hline${ }^{237} \mathrm{~Np}(\mathrm{n}, \mathrm{f})$ & ${ }^{n a t} \mathrm{C}(\mathrm{n}, \mathrm{n})$ \\
\hline${ }^{235,238} \mathrm{U}(\mathrm{n}, \mathrm{f})$ & ${ }^{238} \mathrm{U}\left(\mathrm{n}, \mathrm{n}^{\prime} \mathrm{e}^{-}\right)$ \\
\hline$(n, \gamma)$ Cross sections & ${ }^{48} \operatorname{Ti}\left(\mathrm{n}, \mathrm{n}^{\prime} \gamma\right)$ \\
\hline${ }^{235} \mathrm{U}(\mathrm{n}, \gamma)$ & ${ }^{7} \operatorname{Li}\left(n, n^{\prime} \gamma\right)$ \\
\hline${ }^{242} \mathrm{Pu}(\mathrm{n}, \gamma)$ & ${ }^{233} \mathrm{U}\left(\mathrm{n}, \mathrm{n}^{\prime} \gamma\right)$ \\
\hline${ }^{238} \mathrm{U}\left({ }^{3} \mathrm{He},{ }^{4} \mathrm{He}\right){ }^{237} \mathrm{U},{ }^{238} \mathrm{U}\left({ }^{3} \mathrm{He}, \mathrm{t}\right){ }^{238} \mathrm{~Np},{ }^{238} \mathrm{U}\left({ }^{3} \mathrm{He}, \mathrm{d}\right){ }^{239} \mathrm{~Np}$ & \\
\hline \multicolumn{2}{|c|}{ Decay data } \\
\hline 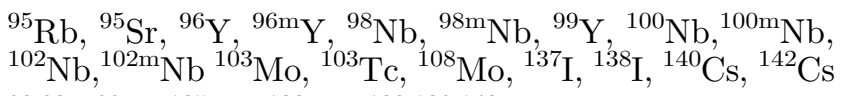 & $\begin{array}{l}\gamma \text { ray and } \beta \text { decay emission probabilities with TAGS } \\
\text { at JYFL }\end{array}$ \\
\hline 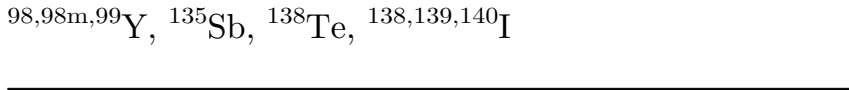 & $\begin{array}{l}\text { Neutron emission probabilities with the BELEN } \\
\text { detector at JYFL }\end{array}$ \\
\hline \multicolumn{2}{|c|}{ Fission yields } \\
\hline${ }^{238} \mathrm{U}(\mathrm{n}, \mathrm{f})$ & Penning trap at JYFL \\
\hline${ }^{233,235} \mathrm{U}(\mathrm{n}, \mathrm{f})$ & Isobaric beams at ILL \\
\hline${ }^{239,241} \mathrm{Pu}(\mathrm{n}, \mathrm{f})$ & Isobaric beams at ILL \\
\hline${ }^{235} \mathrm{U}(\mathrm{n}, \mathrm{f})$ & STEFF spectrometer at $\mathrm{n}_{-}$TOF/EAR2 \\
\hline${ }^{235} \mathrm{U}(\mathrm{n}, \mathrm{f})$ & Orphee reactor at CEA/Saclay \\
\hline${ }^{238} \mathrm{U},{ }^{239} \mathrm{~Np},{ }^{240} \mathrm{Pu},{ }^{244} \mathrm{Cm},{ }^{250} \mathrm{Cf}$ & VAMOS spectrometer at GANIL \\
\hline${ }^{234,235,236,236} \mathrm{U}(\mathrm{g}, \gamma)$ & FRS spectrometer at GSI \\
\hline${ }^{238} \mathrm{U}(\mathrm{n}, \mathrm{f})$ & LICORNE + MINIBALL at IPN/Orsay \\
\hline
\end{tabular}

concentration, and a better INCL-ABLA model by refining the fission modelling.

Publication of results for specialized users and training young scientists: CHANDA scientific activities resulted in over 125 peer reviewed publications, $30 \mathrm{PhD}$ theses and 18 master theses; out of these 48 theses 25 were supported by transnational access and scientific visits to experimental facilities. Also, ERINDA have led to 77 refereed publications and several of the ERINDA supported experiments lead to master and $\mathrm{PhD}$ theses. The transnational access, including user travel support, was instrumental for young researchers to complete their experimental work at stateof-the-art neutron facilities.

The three projects included the support to transnational access to experimental facilities to perform measurements, demonstrations or validations of data, model and methods. The three projects use a similar principle: the simultaneous support to facility and visiting teams together with a review and pooling system as an efficient mechanism to prepare small- and medium-sized experiments. This mechanism has demonstrated to be efficient selecting high-quality experiments and that it helps to use the right facility for each experiment, not just the closest one. The method also provides short reaction time to perform important activities identified during the duration of the project and not identified a priori. Indeed, there were 1 or 2 calls for proposals per year, and that once approved measurements could be started and completed in few months. Interesting examples were measurements at the $n$ TOF EAR2 commissioned during CHANDA and included in the lasts calls for proposals and even the experiments approved at the facility of U. Seville that joined the CHANDA project at the middle of the project.

This mechanism has proven to be very efficient for production of basic research results, demonstration tests, calibration measurements and publications. It is also an efficient education and training tool including $\mathrm{PhDs}$ and master thesis and mobility. In addition, the whole process has helped to improve the facility performance and capabilities, by identifying potential improvements from the request from visiting teams, the suggestions from the evaluation committee, the results of research from the scientific visits of experts and the financial support to compensate the use of the facility. The process also contributes to the facility sustainability for facilities actually used by the ND community, by showing the international needs and also providing part of the operation costs.

As an example of the huge set of results and activities covered by these projects Table 1 lists the main measurements carried out.

\section{Strategic perspectives}

In the preparation of the ND proposals for the 7th EURATOM Framework Program, the ND community used in all cases an inclusive approach, making sure to 
include all EU countries with relevant activities, adding up to 18 countries in CHANDA, also trying to include all institutions with relevant know-how, adding up to 36 institutions, and opening the pooling system for transnational access to all laboratories of potential value, 18 facilities were included in CHANDA.

This process is not simple, as at the same time we have to make sure that each participant has a significant contribution to the project according to their experience and that the effort of the project contribute to improve the high priority nuclear data needs. The process however has been very successful on all the ND projects of FP7 (ANDES, ERINDA and CHANDA) thanks to the interest and goodwill of all the potential partners that acknowledge that putting together this wide collaboration and synchronizing the priorities of the different teams to respond to the EURATOM calls is the most efficient way to be able to address significant challenges at European level and to guarantee the survival of the ND research teams distributed along Europe. Indeed, thanks to this coordination, the relevance, visibility and impact of the European ND research has improved significantly during the last decade and can now compete at the highest world level with initiatives from USA, Russia or Japan.

In this sense, the EURATOM calls and projects have helped to maintain the nuclear data know-how in Europe by aggregation of many and widely distributed small and medium research teams. Efficient collaboration of teams with well identified capacities allows mobilizing the national resources of many teams and becomes a tool for effective addition of resources. Often the problem to organize these collaborations is to prioritize a reduced list of topics for the research, and in this sense the EURATOM calls and projects had been instrumental for the coordination and synchronization by European projects as a way to agree on common priorities. The inclusive approach, needed in all cases to incorporate the required disperse know-how, has allowed avoiding duplication and replacing unnecessary competition with complementarity.

Internal competition both during the preparation of the proposals, by the pooling of the access to facilities and by selection of special actions defined within the project duration had been used to maintain high standards of quality and relevance. This mechanism was reinforced by strong continuous interaction with international bodies managing and discussing the nuclear data activities in the world (NEA/OECD and IAEA) and by an aggressive publication effort.

The resulting ND community participating on the EURATOM projects is a system to develop and maintain the know-how more flexible and effective than large compact teams that has shown to be able to respond efficiently to evolving problems or programs with a large variety of different topics.

Strong coordination and communication of CHANDA, ERINDA, EUFRAT and previously ANDES teams has been reinforced during the whole duration of the EURATOM program, making sure that the transnational access selected could contribute efficiently to the challenges addressed by ANDES or CHANDA. This has also allowed that ERINDA and the TAA of CHANDA contributed to facility improvement and sustainability, and that CHANDA increased the European Nuclear data research community capabilities with upgraded facilities, new detection systems and methods, new tools and in general much better competition and visibility.

\section{Success stories}

Some examples of success stories can be highlighted:

Measuring the same isotope and reaction in two different facilities to reduce systematic effects. For example ${ }^{238} \mathrm{U}$ is a reference isotope and ${ }^{241} \mathrm{Am}[5,6]$ is very difficult to measure because of the high intrinsic radioactivity. Both deserve for different reasons a special effort to reduce the systematic uncertainties. Several sets of measurements using same or similar samples were made for each of these isotopes combining the facilities of GELINA [6] (transmission and capture by $\mathrm{C}_{6} \mathrm{D}_{6}$ ) and $\mathrm{n}_{-} \mathrm{TOF}[5]$ (capture) in this case using 2 different techniques $\left(\mathrm{C}_{6} \mathrm{D}_{6}\right.$ and total absorption calorimeter), the combination of results allows better understanding and qualifying the capture cross section of these isotopes.

With support from ERINDA, CHANDA and OECD/ NEA the GEF code was developed to be a state-of-the-art phenomenological model to give a general description of all fission observables. Results have been included in neutron particle transport codes e.g. MCNP and has led to a highly cited (web of science core collection) publication [7].

Within EUFRAT, studies of $\left(n, n^{\prime} \gamma\right)$ reactions in support to fast reactor developments are carried out at GELINA using the GRAPhEME and GAINS $\gamma$-ray spectrometers. The programme, which is in collaboration with CNRS/IPHC Strasbourg (FR) and IFIN-HH (RO), includes measurements on actinides ${ }^{233} \mathrm{U},{ }^{235} \mathrm{U},{ }^{238} \mathrm{U}$, $\left.{ }^{232} \mathrm{Th}[8,9]\right)$ and light elements $\left({ }^{16} \mathrm{O},{ }^{23} \mathrm{Na},{ }^{28} \mathrm{Si},{ }^{56} \mathrm{Fe}\right)$. At the GAINS spectrometer measurements were carried out to establish a $\gamma$-ray reference cross section for neutroninduced reactions based on the ${ }^{48} \operatorname{Ti}\left(\mathrm{n}, \mathrm{n}^{\prime} \gamma\right)$ and ${ }^{7} \operatorname{Li}\left(\mathrm{n}, \mathrm{n}^{\prime} \gamma\right)$ reactions. The GRAPhEME and GAINS spectrometers will be complemented with an electron spectrometer to study $\left(n, n^{\prime} \gamma\right)$ reactions by the detection of conversion electrons. The development of the DELCO (Detection of Electron from internal Conversion) spectrometer was part of the CHANDA project.

One of the challenges in ND was to propose new experiments in integral and differential facilities based on isotopes of interest for the safety of nuclear systems as well as for their prior known target fabrications difficulties. By having the same origin of fabrication, complementary experiments (integral and microscopic) were proposed and performed within CHANDA to remove the target uncertainties from the comparison. A first test consisted on the pile-oscillation measurements in the MINERVE reactor (CEA) based on Am samples that were manufactured at JRC. This is a first-of-a-kind way of re-using samples that were initially designed for differential measurements at the Geel Van de Graaff, to perform an integral experiment. The experimental results have been used to validate simulation systems based on standard simulation codes for reactor physics and applications: TRIPOLI and MCNP. 
Complementarily within EUFRAT, the transmission and capture cross section measurement stations of GELINA are used to determine neutron-induced interaction cross section data in the resonance region in support to criticality safety analysis in out-of-reactor applications. These studies are part of collaboration with CEA Cadarache (FR), INFN Bologna (IT), IFIN-HH (RO) and ORNL (US). The focus is on fission products with high absorption cross sections, such as Ag [10]. The project includes the characterisation of pellet samples by Neutron Resonance Analysis. The pellets were previously especially prepared for pile oscillator measurements at the MINERVE reactor of CEA Cadarache. These exchanges of samples were proposed within CHANDA. NRA has also been applied to determine the amount of neutron absorbing impurities in material that is used for integral experiments in the VENUS-F facility of the SCK•CEN.

A different success history has been the organization within CHANDA of a network of radioactive samples/ target producers, incorporating within its functions to facilitate the contact between target users and producers and the fabrication capabilities. The network has organized two meetings and has allowed to clarify the requirements from the users and to redefine their request in an efficient manner. This combined with the special extra support foreseen within CHANDA has allowed that from 56 original target requests, 4 were on hold, 7 were cancelled and the remaining 45 were produced and delivered. The list of targets produced included isotopes as ${ }^{7} \mathrm{Be},{ }^{10} \mathrm{Be},{ }^{10} \mathrm{~B},{ }^{13} \mathrm{C}$, ${ }^{44} \mathrm{Ti},{ }^{70,72,73,74,76} \mathrm{Ge},{ }^{91} \mathrm{Nb},{ }^{147} \mathrm{Pm},{ }^{171} \mathrm{Tm},{ }^{204} \mathrm{Tl},{ }^{230} \mathrm{Th},{ }^{233} \mathrm{U}$, ${ }^{235} \mathrm{U},{ }^{237} \mathrm{~Np},{ }^{238} \mathrm{U},{ }^{239} \mathrm{Pu},{ }^{240} \mathrm{Pu},{ }^{241} \mathrm{Am},{ }^{242} \mathrm{Pu}$ and ${ }^{252} \mathrm{Cf}$.

Also deserves a mention, the efficient collaboration setup within the different EURATOM projects for ND in order to join resources to make the best possible global use of the scarce resources available. In this sense, ANDES got support from ERINDA and EUFRAT to perform some of the experiments included in its program. In the case of CHANDA, the functions of ERINDA were already incorporated within CHANDA making even more efficient the integration of measurements and transnational access, but still the collaborations allowed CHANDA to benefit from the support of EUFRAT.

\section{Lessons learnt and remaining challenges}

The most important lessons learnt from the Nuclear Data EURATOM projects are:

- There is a continuous request of new or improved nuclear data that will require supporting R\&D on ND still for many years.

- To be effective the R\&D program on ND has to cover many aspects in a holistic inclusive and comprehensive way.

- Large, widely distributed collaborations, well-coordinated inside inclusive projects, allow performing the required $R \& D$ in an efficient way, maintaining the know-how in Europe by aggregation of many, widely distributed, small and medium research teams.
- The EURATOM financial support allows aggregating these collaborations focussing the research each time around the topics identified on the EURATOM calls, normally well aligned with the high priority request list for nuclear data of the international organizations.

- The EURATOM projects have been very successful to produce the expected results, a large number of publications and $\mathrm{PhD}$ theses and to enhance the relevance and visibility of the European nuclear data R\&D at global level.

Despite the success of CHANDA, several challenges remain for the future:

- Use of the tools developed within CHANDA, ERINDA, EUFRAT and previous projects to deliver more ND needed for safety, industry and society.

- Widen the existing tools to produce data needed for medical and other non-energy applications of ND.

- Reply to new ND needs and continue improving the uncertainty and correlation libraries.

- Validation and verification towards a generic purpose ND library, not as criticality oriented as the present library verification tools.

- Further development and integration of ND know-how in research and final user tools.

- Continue maintaining the know-how in Europe by aggregation of many and widely distributed small and medium research teams.

- Continue supporting the ND facilities and neutron sources.

\section{Impact and possible follow-up actions}

The results of the nuclear data projects, CHANDA, ERINDA and EUFRAT have contributed to the improvement of ND for major isotopes and minor but critical isotopes (for safety, waste management and future concepts) covering the most critical reactions and data needs. These data better enable more reliable simulation and evaluation capabilities that contribute to improve safety and efficiency of the present European reactors. In addition, making available more complete nuclear data and uncertainty libraries help to progress towards best estimate calculation, with an assessment of the final uncertainties on the calculation, to become available for safety assessment, design and operation. All these elements will help to support science-based decision for the energy policies.

Two new nuclear data proposals had been submitted to the EURATOM WP2018. SANDA, with 35 partners, proposing to cover some of the remaining ND challenges after CHANDA and focussed on delivering new data to the end users and to cover energy and non-energy applications, and proposal ARIEL, with 23 partners, to provide transnational access for nuclear data experiments that can be used for training and education in the nuclear field. If they are approved they will probably provide an efficient platform to address the present remaining nuclear data needs at the European Union. 


\section{References}

1. ERINDA: ERINDA web page and the references quoted inside, http://www.erinda.org/

2. EUFRAT: EUFRAT web page, https://ec.europa.eu/jrc/ en/eufrat

3. CHANDA: CHANDA web page and the references quoted, http://www.chanda-nd.eu/

4. M. Barbagallo et al., Be-7(n, alpha)He-4 reaction and the cosmological lithium problem: measurement of the cross section in a wide energy range at nTOF at CERN, Phys. Rev. Lett. 117, 152701 (2016)
5. E. Mendoza et al., Measurement and analysis of the Am-241 neutron capture cross section at the nTOF facility at CERN, Phys. Rev. C 97, 54616 (2018)

6. C. Lampoudis, S. Kopecky, O. Bouland, F. Gunsing, G. Noguere, A.J.M. Plompen, C. Sage, P. Schillebeeckx, R. Wynants, Eur. Phys. J. Plus 128, 86 (2013)

7. K.H. Schmidt et al., General description of fission observables: GEF model code, Nucl. Data Sheets 131, 107 (2016)

8. M. Kerveno et al., Eur. Phys. J. Web Conf. 146, 11012 (2017)

9. M. Kerveno et al., Phys. Rev. C 87, 24609 (2013)

10. L. Śalamon et al., Eur. Phys. J. Web Conf. 146, 11052 (2017)

Cite this article as: Enrique Miguel Gonzalez, Arnd Rudolf Junghans, Arjan Plompen, Peter Schillebeeckx, Nuclear data research supported by EURATOM: CHANDA, ERINDA and EUFRAT, EPJ Nuclear Sci. Technol. 6, 30 (2020) 